\title{
Telessaúde, contextos e implicações no cenário baiano
}

\author{
Telehealth, contexts and implications in Bahia scenario
}

Thiago Gonçalves do Nascimento Piropo' ${ }^{\mathbf{1}}$, Helena Oliveira Salomão do Amaral2

RESUMO O presente texto é um relato de experiência sobre a Telessaúde como estratégia de apoio assistencial, fortalecendo a integração entre os serviços de saúde, ampliando a resolutividade e estabelecendo uma relação custo-efetividade dos processos de saúde pública, além de qualificar Equipes de Saúde da Família através da educação permanente. A Bahia destaca-se por possuir um projeto único no cenário nacional com o objetivo de nortear os projetos intermunicipais, além de mobilizar gestores municipais e profissionais da Atenção Básica, através de treinamento realizado em todas as regiões de saúde. O resultado é ampliação das ações profissionais e acesso a especialidades nos locais mais remotos.

PALAVRAS-CHAVE Telemedicina; Tecnologia da informação; Saúde pública; Atenção Primária à Saúde.

ABSTRACT The present text is a report on the experience of Telehealth as a strategy of assistance support, strengthening the integration between health services, increasing the resolution and establishing a cost-effectiveness relation of procedures regarding public health, besides qualifying Family Health Teams through continuing education. Bahia is distinguished for having a single project in the national scene aiming to guide the intermunicipal projects, in addition to mobilizing Primary Care professionals and city managers through training conducted in all of the health regions. The result is the extension of professional actions and access to medical specialties in the most remote locations.

KEYWORDS Telemedicine; Information technology; Public health; Primary Health Care.

${ }^{1}$ Secretaria Estadual de Saúde da Bahia (Sesab), Núcleo de Tecnologia de Informação e Comunicação em Saúde (Nutics) Salvador (BA), Brasil. piropo.t@gmail.com 


\section{Introdução}

Os avanços tecnológicos contemporâneos vêm sendo incorporados às diversas vertentes sociais e trazendo importantes contribuições na prestação da assistência qualificada. Tais avanços têm como elementos centrais um conjunto de tecnologias sustentadas por fundamentos na microeletrônica, nas telecomunicações e na informática, denominada Tecnologia da Informação e Comunicação (TIC) (IBGE, 2009).

Para a Organização Mundial de Saúde (OMS), as tecnologias de informação e comunicação corroboram com a prestação de cuidados na saúde pública, pesquisas e atividades relacionadas à saúde para o benefício, principalmente, de países em desenvolvimento (OMS, 2012). Permite, ainda, a instituição de ferramentas estratégicas de apoio ao desenvolvimento das ações em saúde no que se refere ao planejamento, assistência, pesquisa e educação em saúde (NOVAES, 2010).

A principal expectativa referente à assistência na saúde coletiva, considerada pela OMS (2009), é a melhoria do acesso aos recursos de melhor qualidade disponíveis na área de saúde para a maior parte da população mundial. Considera, também, a incorporação de tecnologias como um instrumento político e estratégico no planejamento e na execução de ações em saúde.

Segundo Santos (2006), a necessidade de atuação em cenários cada vez mais amplos e, ao mesmo tempo, cheios de possibilidades imprime ao processo de trabalho na área da saúde desafios constantes. Estabelecer uma melhor relação custo-efetividade dos processos de saúde pública e permitir que as facilidades dos grandes centros cheguem aos locais mais remotos são alguns dos obstáculos a serem superados (SANTOS, 2006). Nesse contexto, o conceito de Telessaúde surge como uma ferramenta potencializadora para os serviços de saúde.

O termo 'Telessaúde' (ou telemedicina, e-Saúde) vem sendo usado para designar as atividades que utilizam as tecnologias de informação e comunicação na atenção à saúde
(NORRIS, 2002). Baseia-se, portanto, na telemática, podendo ser definida como uma ciência que estuda a manipulação e a utilização da informação através do uso combinado de computador, seus acessórios e meios de comunicação.

O primeiro termo utilizado para designar ações de saúde a distância foi a telemedicina, caracterizada pela transferência de informações médicas em processos de diagnóstico, terapêutica e educação através do uso da tecnologia de informação e telecomunicação (AMERICAN TELEMEDICINE ASSOCIATION, 2006). Tem por finalidade a prestação de apoio clínico, objetivando melhores resultados de saúde, superando barreiras geográficas e conectando os usuários que não estão no mesmo local físico a partir da utilização de vários tipos de TIC (OMS, 2009).

Silva (2012), entretanto, considera o termo 'telemedicina' limitado frente a sua aplicabilidade nos campos da saúde, tecnologia e informação por apreciar que as atividades da área da saúde não são de exclusividade médica. A Telessaúde, portanto, traz referências mais amplas e é definida como o "uso das tecnologias de informação e comunicação para transferir informações de dados e serviços clínicos, administrativos e educacionais em saúde" (NORRIS, 2002, P. 177). Abrange, ainda, os profissionais de saúde em geral, incluindo médicos, enfermeiros, fisioterapeutas, farmacêuticos, técnicos, entre outros.

Para Novaes (2010), o termo 'Telessaúde' é definido como o uso das modernas tecnologias da informação e comunicação para atividade a distância relacionada à saúde em seus diversos níveis (primário, secundário e terciário), possibilitando a interação entre profissionais de saúde ou entre estes e seus pacientes, bem como o acesso remoto a recursos de apoio diagnósticos ou até mesmo terapêuticos através da robótica.

Já o termo 'e-Saúde' (eHealth) permanece confuso na literatura. Pode ser caracterizado não somente como um desenvolvimento da tecnologia, mas também como uma nova forma de trabalho, uma atitude e um relacionamento via acesso em rede, uma forma de pensamento 
globalizado, com o objetivo de promover o cuidado em saúde local, regional e mundial por meio do uso da tecnologia de informação e comunicação (NORRIS, 2002).

A OMS o define como

a utilização, pela área de saúde, de dados digitais que são transmitidos, armazenados e recuperados eletronicamente e que podem ser usados no apoio ao serviço de assistência médica a distância ou em seu próprio local. (2012, P. 05).

Apoia, ainda, a prestação de serviços de saúde e a gestão dos sistemas de saúde.

A aplicabilidade do uso da Telessaúde é diversa, considerando-se as necessidades e ferramentas envolvidas. No contexto da Atenção Básica (AB), a Portaria $\mathrm{n}^{0}$ 2.546, de 27 de outubro de 2011, do Ministério da Saúde (MS), versa sobre alguns serviços disponíveis aos profissionais e trabalhadores das Redes de Atenção à Saúde (RAS) no Sistema Único de Saúde (SUS) e define Teleconsultorias, Segunda Opinião Formativa, Tele-educação e Telediagnósticos.

Em seu Art. $2^{\circ}$, a Portaria GM/MS 2.546/11 (BRASIL, 2011) define teleconsultoria como:

[...] consulta registrada e realizada entre trabaIhadores, profissionais e gestores da área de saúde, por meio de instrumentos de telecomunicação bidirecional, com o fim de esclarecer dúvidas sobre procedimentos clínicos, ações de saúde e questões relativas ao processo de trabalho [...].

A teleconsultoria pode ser realizada de duas maneiras: em tempo real, chamada de teleconsultoria síncrona, geralmente realizada por chat, web ou videoconferência; ou ainda assíncrona, quando é realizada por meio de mensagens off-line. Esta última é a mais frequente e comumente envolve assuntos pertinentes à Atenção Primária à Saúde (APS), permitindo, ainda, o requerimento de materiais de leitura/aprendizagem, teórico ou teórico-prático sobre um assunto específico para o desenvolvimento de alguma atividade de interesse (BRASIL, 2012).
As demandas por teleconsultorias síncronas, em geral, envolvem casos clínicos de pacientes ou questões de saúde mais complexas e interdisciplinares e ocorrem em tempo real, após agendamento prévio, através de uma webconferência (BRASIL, 2012).

A mesma portaria define a Segunda Opinião Formativa (SOF) como:

[...] resposta sistematizada, construída com base em revisão bibliográfica, nas melhores evidências científicas e clínicas e no papel ordenador da Atenção Básica à saúde, a perguntas originadas das teleconsultorias, e selecionadas a partir de critérios de relevância e pertinência em relação às diretrizes do SUS. (BRASIL, 2012, P. 36).

Para o MS (BRASIL, 2012), caso o teor da resposta de uma teleconsultoria assíncrona seja pertinente e replicável nos contextos regionais e/ou nacional de fissionais em uma SOF, será disponibilizada pela Biblioteca Virtual em Saúde (BVS). A resposta deve ser sistematizada, construída com base em revisão bibliográfica, nas melhores evidências científicas e clínicas e no papel ordenador da Atenção Básica à saúde, na observância às diretrizes do SUS.

A BVS é uma das ferramentas de educação permanente, entre inúmeras possibilidades de qualificação profissional que a Telessaúde oferece a partir de Educação a Distância (EAD), denominada como tele-educação. Para o MS (BRASIL, 2012), trata-se de uma forma de ensino que permite a aprendizagem, com a mediação humana de recursos didáticos sistematicamente organizados, apresentados em diferentes suportes de informação, utilizados isoladamente ou combinados, e veiculados pelos diversos meios de comunicação.

Outro serviço utilizado na prática clínica é o telediagnóstico, definido pelo MS através da Portaria $n^{\circ} 2.546$ como uma oferta autônoma que utiliza as tecnologias de informação e comunicação de modo a embasar o diagnóstico através de distâncias 
geográficas e temporais (BRASIL, 2011). A implantação de serviços de telediagnóstico possibilita a redução dos custos em saúde, promovendo acessibilidade a diagnósticos e tratamentos oportunos, por evitar longos deslocamentos de pacientes e profissionais. Permite, contudo, que o exame complementar seja coletado no âmbito da APS e tenha seu laudo realizado em centros de referência, sem a necessidade da presença do médico especialista no momento da execução do exame (OLIVEIRA, 2010; BRASIL, 2011).

Historicamente, Zundel (1996) relata o primeiro registro do uso de tecnologias para realização de testes diagnósticos em 1950 a partir de um aparelho de fax, em que foi possível a transmissão de um Raio-X para serviços especializados em radiologia nos Estados Unidos. Porém, com os avanços das tecnologias de telecomunicação, mais evidentes na década de 1990, as dificuldades de acesso a exames em áreas remotas foram aos poucos sendo superadas (BRASIL, 2011).

Os cenários para aplicabilidade dos serviços de Telessaúde são diversos, entretanto, objetivando melhorar a qualidade da assistência prestada, permitir o acesso a serviços especializados e aumentar a resolutividade do nível básico, entre outros, as zonas rurais e comunidades situadas em regiões mais remotas são as principais beneficiadas com a implantação da Telessaúde (SANTOS, 2006).

Para o sucesso do programa, é fundamental o envolvimento da comunidade e do poder público na identificação de áreas e populações que apresentem agravos de saúde e/ou deficiência na assistência. A partir de um levantamento técnico capaz de apontar os reais problemas de saúde, é possível adequar as condutas à realidade local e às condições culturais e socioeconômicas da população a ser beneficiada pelos serviços advindos da implantação da Telessaúde (SANTOS, 2006; SILVA; MORAES, 2012).

O SUS preconiza a universalização do acesso, integralidade da atenção, equidade, descentralização da gestão, hierarquização dos serviços e controle social na construção e pactuação da política de saúde, o direito a mesma, prezando pela prevenção e promoção da saúde. São inegáveis os avanços do SUS no Brasil nos últimos anos, no entanto, diante de novos desafios, se faz necessária a instituição de estratégias capazes de abranger primordialmente as populações excluídas.

Alguns estudos têm apontado dificuldades de acesso a serviços de saúde em comunidades remotas, a exemplo da população quilombola no sudoeste baiano. A iniquidade no direito à saúde vai além das limitações locais, sendo expressas, sobretudo, pelas precárias condições sociais e econômicas. O mesmo caráter limitante é aplicado à realidade de populações de zonas rurais, onde é imperativa a adaptação da dinâmica do processo de trabalho dos programas de saúde, e estratégias para permitir a acessibilidade e a utilização destes serviços, considerando a cultura local, costumes e prevalência das enfermidades (GOMES ET AL., 2013).

O estudo de Soares e Coelho (2008) sobre cuidados infantis em comunidades rurais na Bahia, de um modo geral, referenciou as dificuldades no acesso aos serviços de saúde enfrentados pelas famílias estudadas. Assis et al. (2007) faz alusão às desigualdades sociais nos acessos aos serviços de saúde, com capacidade resolutiva no nordeste brasileiro, aliadas às precárias condições de vida, disparidades econômicas, deficiência nutricional, principalmente nos infantes, e precárias condições sanitárias.

Esse cenário, contudo, fortalece a necessidade iminente da fixação de políticas públicas de universalização do acesso aos serviços de saúde a grupos populacionais longínquos, sendo a Telessaúde, segundo Santos et al. (2006), uma alternativa viável capaz de propiciar melhorias na qualidade da assistência prestada.

Na Bahia, a Telessaúde teve início em 2011, entretanto, obteve maior visibilidade a partir de 2013, quanto ocorreu aumento significativo na adesão dos profissionais de saúde, entendendo o potencial que esse instrumento 
representa na qualificação de suas ações junto as Equipes de Saúde da Família (EqSF), superando as distintas características geográficas em todo o território baiano.

\section{Caracterizando o cenário baiano}

O Estado da Bahia está localizado na região Nordeste, onde ocupa uma área de 564.733 km². É caracterizado por planície litorânea, planaltos, chapadas, serras e depressões. Possui uma população de 14.016.906 de habitantes, segundo o último censo de 2010, dispostos entre os 417 municípios, apresentando densidade demográfica de 24,82 (hab/ $\left.\mathrm{km}^{2}\right)$ (IBGE, 2010).

Tais características vêm sendo referidas em alguns estudos, correlacionando-as com as ações de saúde. Cunha, Silva e Maria (2010) relataram sobre acessibilidade aos serviços de saúde em um município baiano abordando a relação da organização dos serviços com os aspectos geográficos, em alusão à distribuição espacial dos recursos, à existência de transporte e à localização das Unidades Básicas de Saúde (UBS). Oliveira et al. (2012) corrobora com Cunha, Silva e Maria (2010) ao apontar os fatores geográficos como importantes obstáculos para o acesso aos serviços de saúde.

Em outro estudo, foi observado por Silva et al. (2011) que para a inserção de uma UBS é necessário conhecer as múltiplas territorialidades existentes em um espaço geográfico, a fim de facilitar a acessibilidade ao serviço de saúde para não comprometer o cuidado e a continuidade dos tratamentos e reflexos negativos na população.

A AB na Bahia, contudo, caracteriza-se por estar presente em $99 \%$ dos municípios, com 2.894 EqSF implantadas. Possui cobertura populacional estimada de $66,85 \%$ - aproximadamente 9 milhões e 300 mil pessoas beneficiadas pela Estratégia Saúde da Família. Essa cobertura baseia-se no cadastro da população da área de abrangência de atuação das EqSF no Sistema de Informação da Atenção Básica (Siab). Ainda, 43,55\% das internações na rede do SUS são motivadas por condições sensíveis à APS (BAHIA, 2013).

Como forma de fortalecer as ações da $\mathrm{AB}$, o projeto nacional intitulado Telessaúde Brasil Redes foi reformulado para atender às necessidades baianas, considerando suas particularidades sanitárias e epidemiológicas presentes nas nove macrorregiões de saúde (Sul, Sudoeste, Extremo-Sul, Norte, CentroNorte, Nordeste, Oeste, Leste e Centro-Leste). Isto posto, a Secretaria da Saúde do Estado (Sesab) apresentou o projeto único intitulado Projeto Telessaúde Brasil Redes - Bahia.

\section{O projeto único de Telessaúde Brasil Redes como diferencial de implantação}

O Projeto Telessaúde Brasil Redes - Bahia foi construído pela Sesab, em parceria com outras instituições, baseado na proposta da Secretaria de Gestão do Trabalho e da Educação na Saúde (SGTES) do MS, em julho de 2011 (Portaria 402/2010), aprovado pela Resolução CIB BA $\mathrm{N}^{\mathrm{o}} 143 / 2011$ de 21 de Julho de 2011 e aprovado pela SGTES no mesmo ano (BRASIL, 2011).

Em função da Portaria GM/MS $n^{\circ}$ $2.554 / 2011$, houve a abertura de sistema de cadastro de propostas intermunicipais e estaduais, em novembro de 2011, sob coordenação do Departamento de Atenção Básica (DAB) do MS. O Conselho Estadual dos Secretários Municipais de Saúde (Cosems) e a Sesab propuseram como critério que fossem cadastrados todos os municípios prioritários para a Rede Cegonha (pertencentes às Macrorregiões Leste, Centro-Norte, Norte e Sul da Bahia) e todas as equipes dos 100 municípios do projeto supracitado aprovado pela SGTES/MS.

Foram, então, cadastradas cinco propostas intermunicipais, considerando como Núcleos Técnico-Científicos cinco municípios destas regiões prioritárias - Juazeiro, Capim Grosso, Vera Cruz, Porto Seguro e Itabuna - e 
uma proposta estadual considerando a Sesab como Núcleo Técnico-Científico.

Durante o cadastro de tais propostas, foi verificado que não havia como vincular cada município a sua macrorregião de assistência em saúde, segundo o Plano Diretor de Regionalização (PDR), pois iria ultrapassar o teto fornecido pelo sistema. Dessa forma, a fim de garantir o envio das propostas, os pontos de Telessaúde de 356 municípios foram alocados aleatoriamente nas propostas intermunicipais, com a perspectiva de reordenação desses pontos após a aprovação dos projetos pelo MS.

Considerando, portanto, como diretriz o projeto único Telessaúde desenvolvido na Bahia, em janeiro de 2012, foi aprovada em Comissão Intergestores Bipartite da Bahia a construção do Projeto Estadual que nortearia todos os Núcleos Técnico-Científicos, bem como o uso do financiamento de duas fontes do MS (SGTES e DAB) para o Telessaúde.

Este projeto único, de Telessaúde Brasil Redes no Estado da Bahia, é resultante de parcerias entre a Sesab, Cosems, Secretarias Municipais de Saúde, Universidade Federal da Bahia (UFBA) e Fundação Estatal Saúde da Família (Fesf-SUS). Nessa conformação, a Bahia tem um núcleo único, denominado Núcleo Técnico-Científico (NTC) da Bahia, característica que o diferenciou entre os núcleos implantados até então pelos demais estados brasileiros.

A Fesf-SUS, mediante contratualização com a gestão estadual e as municipais que tiveram propostas intermunicipais aprovadas pelo MS, tem ofertado os serviços de Telessaúde do NTC da Bahia, e por meio desse novo projeto foi possível a ampliação dos pontos no Estado e a ampliação da equipe do NTC capaz de atender às demandas dos novos pontos de Telessaúde.

Desse modo, dos 417 municípios baianos, 296 receberam repasses financeiros fundo a fundo para a estruturação de Pontos de Telessaúde. A construção do projeto único permitiu a integração de todos os municípios, ficando a Sesab com o compromisso de distribuir 759 computadores às equipes que ainda não haviam sido contempladas com pontos de Telessaúde.

Em 2013, a diretoria de AB da Sesab contou com 60 sanitaristas que executaram o apoio institucional para todos os municípios baianos. Em oficinas de acolhimento com profissionais da saúde e rodas de coordenadores da $\mathrm{AB}$, os apoiadores sanitaristas puderam abordar a Telessaúde com gestores e trabalhadores, ação configurada como importante estratégia para o momento de implantação.

As ações do NTC da Bahia contaram com uma equipe de 22 profissionais em sua formação inicial, com perspectiva de ampliação, e desenvolveram atividades nos 31 municípios sede de Diretorias Regionais de Saúde (Dires), com o objetivo de apresentar o projeto, os serviços disponíveis, além de realizar o treinamento na plataforma online do MS.

A atuação dos técnicos das Dires foi fundamental para ampliar a mobilização dos profissionais dos municípios. Todos os esforços resultaram na participação de 2.612 profissionais do campo da $\mathrm{AB}$ de $386 \mathrm{mu}-$ nicípios, totalizando 534 horas de trabalho dedicadas às oficinas de capacitação.

As oficinas foram divididas em duas etapas: a primeira consistiu na exposição da parte teórica referente ao histórico do projeto, apresentação da equipe do NTC, dos serviços ofertados e da plataforma online, seguida por uma aula prática, na qual os profissionais da $\mathrm{AB}$ operaram e registraram solicitações de teleconsultorias. Ao final de cada turma de profissionais, um instrumento de avaliação foi entregue aos participantes, que responderam, anonimamente, aos itens referentes à metodologia aplicada e entendimento da Telessaúde.

Ao observarmos o escopo dessas avaliações, é possível vislumbrar cenários de aproveitamento do TelessaúdeBA no cotidiano ambulatorial dos profissionais da saúde. Para os participantes das oficinas, o programa irá apoiar o trabalho das EqSF, sobretudo, em municípios distantes e com pouca infraestrutura. Além disso, eles acreditam que será um instrumento para pesquisa, estudo e 
educação, facilitando o retorno em relação às informações de saúde dos pacientes, além de gerar subsídios que fortalecem a confiança da unidade de saúde, bem como entre os profissionais da equipe como um todo (BAHIA, 2013).

As oficinas se constituíram como a primeira fase de implantação do projeto, chamada de I Ciclo de Treinamentos Microrregionais de Profissionais da Atenção Básica para Uso dos Serviços do TelessaúdeBA. Os resultados obtidos superaram a meta estabelecida inicialmente, que era de 70\% das EqSF cadastradas em toda a Bahia, conforme dados consolidados apresentados em planilhas de monitoramento institucionais.

No período compreendido entre 12 de julho de 2013 e 27 de junho de 2014 - último levantamento feito pelos administradores da plataforma -, foram registradas 2.530 solicitações de teleconsultorias (síncronas e assíncronas), respondidas, de maneira compartilhada, pela equipe de teleconsultores do Núcleo da Bahia e do Rio Grande do Sul (TelessaúdeRS) e por 10.847 profissionais da $\mathrm{AB}$ cadastrados.

Além das teleconsultorias, o TelessaúdeBA conta com 20 pontos de Telediagnóstico em Cardiologia implantados e situados em municípios distintos no Estado. Ao todo, 7.983 laudos de Eletrocardiograma (ECG) já foram entregues até abril de 2014, bem como a elaboração de SOF pelo núcleo baiano, disponíveis no site da BVS do MS.

A figura 1 mostra o consolidado dos dados do TelessaúdeBA já descritos e obsevados no período compreendido entre julho de 2013 e junho de 2014.

Figura 1. O cenário baiano: consolidado dos dados do TelessáudeBA

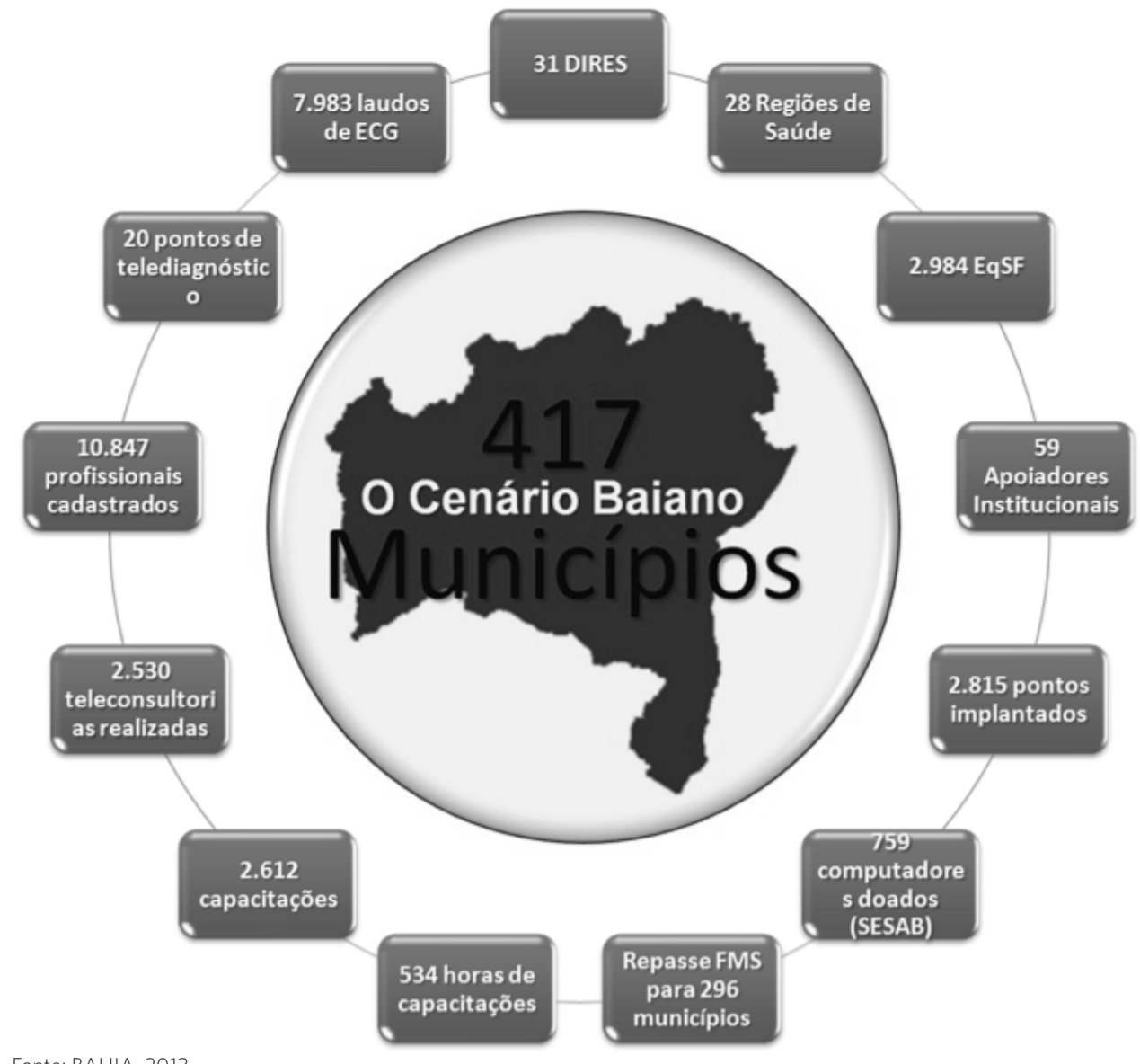

Fonte: BAHIA, 2013 


\section{Considerações finais}

A superação das barreiras físicas e geográficas a partir do uso de tecnologia em práticas de saúde torna essa ferramenta valiosa e indispensável para formação, atualização e ampliação da prática profissional em saúde. Em um estado com grande extensão territorial como a Bahia, a utilização de tecnologias de comunicação pode auxiliar a diminuir as desigualdades observadas.

A utilização dos serviços da Telessaúde, e outras terminologias afins, emergem como novos instrumentos significativos para transpor as barreiras culturais, socioeconômicas e geográficas para os serviços e informação em saúde, principalmente na assistência remota e disseminação de informação, evitando a locomoção para os centros de referência.

Dessa forma, a Telessaúde pode ampliar as ações de profissionais afins, integrando-os aos serviços de saúde locais e, convenientemente, aos serviços localizados em hospitais e centros de referência, mantendo um mecanismo de atendimento contínuo para prevenção, diagnóstico e tratamento.

Em uma perspectiva de melhoria da assistência em saúde, os serviços a distância se configuram como uma excelente oportunidade, principalmente para as regiões carentes e remotas, onde não se pode esperar uma cobertura especializada presencial. A aplicação de tecnologias de baixo custo, portanto, se faz necessária e deve ser estimulada de forma sustentável, de modo a consolidar redes colaborativas integradas de assistência à saúde a distância.

As práticas de Telessaúde vêm crescendo em todo o País, ampliando as discussões que devem ser pautadas em critérios éticos e legais, favorecendo o aumento de publicações do setor. Para isso, o envolvimento dos profissionais, o engajamento dos Conselhos Federal, Regionais e da sociedade científica são essencialmente importantes na condução das discussões que pautam a atuação das ações da Telessaúde como instrumento transformador.

\section{Referências}

\section{AMERICAN TELEMEDICINE ASSOCIATION. \\ Telemedicine: a brief overview, congressional Telehealth briefing, Washington, DC. 1999. Disponível em: <http://www.atmeda.org/news/overview.html>. Acesso em: 20 out. 2013.}

ASSIS, A. M. O. et al. Desigualdade, pobreza e condições de saúde e nutrição na infância no Nordeste brasileiro. Cad. Saúde Pública, Rio de Janeiro, v. 23, n. 10, p. 2337-2350, 2007. Disponível em: <http://www.scielosp. org/pdf/csp/v23n10/09.pdf>. Acesso em: 20 out. 2013.

BAHIA. Secretaria Estadual de Saúde da Bahia. Diretoria de Atenção Básica. Coordenação de Avaliação e Monitoramento da Atenção Básica. 2013. Disponível em: <http://www.saude.ba.gov.br/dab>.
Acesso em: 28 out. 2013.

BRASIL. Ministério da Saúde. Manual de Telessaúde para Atenção Básica/Atenção Primária à Saúde. Brasília, DF: Ministério da Saúde, 2012. (Série A. Normas e Manuais Técnicos). Disponível em: <http:// telessaude.bvs.br/tiki-download_file.php?fileId=2450>. Acesso em: 01 nov. 2013.

Ministério da Saúde. Gabinete do Ministro. Portaria $n^{\circ} 2.546$, de 27 de outubro de 2011. Redefine e amplia o Programa Telessaúde Brasil, que passa a ser denominado Programa Nacional Telessaúde Brasil Redes (Telessaúde Brasil Redes). Brasília, DF, 2011. Disponível em: <http://www.brasilsus.com.br/legislaco-es/gm/110256-2546.html>. Acesso em: 01 nov. 2013. 
CUNHA, A. B. O.; SILVA, V.; MARIA, L. Acessibilidade aos serviços de saúde em um município do Estado da Bahia, Brasil, em gestão plena do sistema. Cad. Saúde Pública, Rio de Janeiro, v. 26, n. 4, p. 725-737, 2010. Disponível em: <http://repositorio.ufba.br/ri/handle/ ri/2399>. Acesso em: 21 out. 2013.

GOMES, K. O. et al. Utilização de serviços de saúde por população quilombola do Sudoeste da Bahia, Brasil. Cad. Saúde Pública, Rio de Janeiro, v. 29, n. 9, p. 1829-1842, 2013. Disponível em: <http://www.scielo.br/pdf/csp/ v29n9/a22v29n9.pdf >. Acesso em: 20 out. 2013.

\section{INSTITUTO BRASILEIRO DE GEOGRAFIA E} ESTATÍSTICA (IBGE). Censo 2010. [internet]. Disponível em: <http://www.ibge.gov.br/estadosat/>. Acesso em: 20 out. 2013.

Diretoria de Pesquisa. O setor de Tecnologia da Informação e Comunicação no Brasil 2003-2006. Rio de Janeiro: IBGE, 2009. (Estudos e pesquisas, informação econômica, 11).

NORRIS, A. C. Essentials of Telemedicine and Telecare. Baffins Lane: John Wiley \& Sons, 2002.

NOVAES, M. A. et al. A Telessaúde na Estratégia Saúde da Família em Pernambuco: resultados e desafios. In: CONGRESSO BRASILEIRO DE INFORMÁTICA EM SAÚDE. Recife. 2010. Disponível em: <http://www. cbtms.org.br/congresso/trabalhos/052.pdf $>$. Acesso em: 01 nov. 2013.

OLIVEIRA, D. G. Análise do grau de implantação da Telessaúde na Estratégia Saúde da Família em Pernambuco: estudo de casos. 2010. 124 f. Dissertação (Mestrado em Saúde Pública) - Centro de Pesquisas Aggeu Magalhães, Fundação Oswaldo Cruz, 2010. Disponível em: <http://www.cpqam.fiocruz.br/ bibpdf/2010oliveira-dg.pdf>. Acesso em: 02 nov. 2013.

OLIVEIRA, L. S. et al. Acessibilidade a atenção básica em um distrito sanitário de Salvador. Ciênc. saúde coletiva, v. 17, n. 11, p. 3047-3056, 2012. Disponível em: <http://www.scielosp.org/scielo.php?script=sci arttext\&pid $=$ S1413-81232012001100021 $>$. Acesso em: 28 out. 2013.
ORGANIZAÇÃO MUNDIAL DA SAÚDE (OMS). National eHealth strategy toolkit. 2012. Disponível em: $<$ http://www.itu.int/dms_pub/itu-d/opb/str/D-STR-E_HEALTH.05-2012-PDF-E.pdf>. Acesso em: 05 out. 2013.

ORGANIZAÇÃO MUNDIAL DA SAÚDE (OMS). Telemedicine, opportunities and developments in

Member States: report on the second global survey on e-Health. 2009. Disponível em: <http://www.who.int/ goe/publications/ehealth_series_vol2/en/>. Acesso em: 11 out. 2013.

SANTOS, A. F. et al. Telessaúde: um instrumento de suporte assistencial e educação permanente. Belo Horizonte: UFMG, 2006.

SILVA, A. B.; MORAES, I. H. S. O caso da Rede Universitária de Telemedicina: análise da entrada da Telessaúde na agenda política brasileira. Physis: Revista de Saúde Coletiva, Rio de Janeiro, v. 22, n. 3, p. 1211-1235, 2012. Disponível em: <http://www.scielo.br/pdf/physis/v22n3/19.pdf>. Acesso em: 20 out. 2013.

SILVA, L. O. L. et al. Acessibilidade ao serviço de saúde: percepções de usuários e profissionais de saúde. Cogitare enferm., Salvador,v. 16, n. 4, p. 654-660, 2011. Disponível em: <https://repositorio.ufba.br/ri/handle/ ri/2399>. Acesso em: 28 out. 2013.

SOARES, M. D.; COELHO, T. C. B. O cotidiano do cuidado infantil em comunidades rurais do Estado da Bahia: uma abordagem qualitativa. Rev. Bras. Saúde Mater. Infant., Recife, v. 8, n. 4, p. 463-472, 2008. Disponível em: <http://www.scielo.br/pdf/rbsmi/ v8n4/12.pdf $>$. Acesso em: 20 out. 2013.

ZUNDEL, K. M. Telemedicine: history, applications, and impact on librarianship. Bulletin of the Medical Library Association, Chicago, v. 84, n. 1, p. 71-79, 1996.

Recebido para publicação em julho de 2014

Versão final em janeiro de 2015

Conflito de interesses: inexistente

Suporte financeiro: não houve 